\title{
Feldenkrais-Methode - Bewusst motorische Ineffizienz beheben
}

In der Mitte des 20. Jahrhunderts entwickelte der israelische Kernphysiker Dr. Moshé Feldenkrais auf der Grundlage eigener körperlicher Beschwerden und inspiriert durch die Kampfsportarten Judo und Jiu-Jitsu seine Feldenkrais-Methode. Hierin betont er die wechselseitige Beziehung unseres Muskel- und Nervensystems. Diese beiden Systeme hängen ihrerseits sehr eng zusammen mit der Schwerkraft, die als Gegenkraft beim Aufrichten des menschlichen Körpers wirkt. So sind die erlernten Bewegungsmuster selten die einfachsten oder ökonomischsten und führen daher oft zu körperlichen Problemen, so Dr. Feldenkrais. Unsere große „Verwundbarkeit“ resultiere aus unserem fortgeschrittenen evolutionären Entwicklungsstand im Vergleich zur Tierwelt, in der Bewegung eine nebensächliche Rolle spielt. Die FeldenkraisMethode versucht nun, diese motorische Ineffizienz zu beheben, indem die strukturierten Bewegungen bewusst und auf leichteste Art ausgeführt werden und damit den Bewegungssinn schulen. „Erst wenn wir wissen, was wir tun, können wir tun, was wir wollen “, so Dr. Mosche Feldenkrais (Paschen 2006). Die Feldenkrais Methode basiert also, ähnlich wie die Pilates Methode und die Alexandertechnik, auf das Lernen Bewegung bewusst wahrzunehmen. Anfangs sind die Bewegungsübungen klein und einfach, werden allmählich größer und komplexer. Durch motorische Lernprozesse soll die Qualität der Bewegungen verbessert und verfeinert werden. Recherche in der Datenbank für evidenzbasierte Physiotherapie PEDRO und der Datenbank für biomechanische Literatur und wissenschaftlichen Studien PubMed konnte auch für die Feldenkrais Methode keine überzeugende Effektivität nachweisen. 